\section{ETHNIC DIFFERENCES IN PHENOTYPIC EXPRESSION OF HYPERTROPHIC CARDIOMYOPATHY}

doi:10.1136/heartjnl-2011-300198.170

N Sheikh, M Papadakis, N Chandra, H Raju, A Zaidi, S Ghani, M Muggenthaler, S Gati, S Sharma. St. George's University of London, London, UK

Purpose Hypertrophic Cardiomyopathy is a heterogeneous condition with variable phenotypic expression. Current studies are based on predominantly Caucasian cohorts (white patients; WP), therefore the phenotypic manifestations of HCM in individuals of African/Afro-Caribbean origin (black patients; BP) are not fully realised. Data in athletes and hypertensive patients indicate that black ethnicity is associated with a greater prevalence of repolarisation abnormalities on the ECG as well as a greater magnitude of left ventricular hypertrophy (LVH), highlighting the importance of defining the HCM phenotype in this ethnic group.

Methods Between 2001 and 2010, 155 consecutive patients with HCM (52 BP, 103 WP) were assessed in 3 specialist cardiomyopathy clinics in South London. All individuals underwent comprehensive

\section{Abstract 170 Table 1}

\begin{tabular}{|c|c|c|c|}
\hline & $\begin{array}{l}\text { Black HCM } \\
(n=52)\end{array}$ & $\begin{array}{l}\text { white HCM } \\
(n=103)\end{array}$ & p Value \\
\hline \multicolumn{4}{|l|}{ Demographics } \\
\hline Age of diagnosis (years) & $48.1 \pm 17.1$ & $50.5 \pm 16.5$ & 0.552 \\
\hline Gender (males) & $61.5 \%$ & $62.1 \%$ & 0.942 \\
\hline $\mathrm{FH}$ of $\mathrm{HCM} / \mathrm{SCD}$ & $34.6 \%$ & $32.0 \%$ & 0.747 \\
\hline NYHA functional class III or IV & $7.7 \%$ & $7.8 \%$ & 0.987 \\
\hline Patients on treatment & $55.8 \%$ & $46.6 \%$ & 0.281 \\
\hline B-blockers & $28.8 \%$ & $39.1 \%$ & 0.445 \\
\hline Calcium antagonists & $26.9 \%$ & $12.6 \%$ & 0.026 \\
\hline Amiodarone & $7.7 \%$ & $1.9 \%$ & 0.080 \\
\hline Diuretics & $13.5 \%$ & $9.7 \%$ & 0.480 \\
\hline Disopyramide & $3.8 \%$ & $9.7 \%$ & 0.197 \\
\hline Intracardiac defibrillator in situ & $5.8 \%$ & $5.8 \%$ & 0.989 \\
\hline \multicolumn{4}{|l|}{ Echocanliographic characteristics } \\
\hline Ao (mm) & $31.3 \pm 3.7$ & $33.2 \pm 5.8$ & 0.123 \\
\hline $\mathrm{LA}(\mathrm{mm})$ & $40.9 \pm 7.3$ & $39.9 \pm 7.3$ & 0.593 \\
\hline LVED (mm) & $44.0 \pm 6.1$ & $44.4 \pm 6.1$ & 0.787 \\
\hline mLVWTd (mm) & $17.3 \pm 4.9$ & $18.8 \pm 4.1$ & 0.069 \\
\hline LV mass $(\mathrm{g})$ & $279.6 \pm 106.5$ & $287.6 \pm 112.7$ & 0.767 \\
\hline FS (\%) & $40.4 \pm 9.1$ & $39.8 \pm 8.3$ & 0.641 \\
\hline E wave $(\mathrm{m} / \mathrm{s})$ & $0.70 \pm 0.18$ & $0.74 \pm 0.20$ & 0.443 \\
\hline A wave $(\mathrm{m} / \mathrm{s})$ & $0.67 \pm 0.18$ & $0.66 \pm 0.27$ & 0.851 \\
\hline$E / A$ & $1.11 \pm 0.44$ & $1.22 \pm 0.58$ & 0.422 \\
\hline SAM & $23.1 \%$ & $37.9 \%$ & 0.064 \\
\hline LVOT gradient $=30 \mathrm{~mm} \mathrm{Hg}$ & $23.1 \%$ & $34.0 \%$ & 0.163 \\
\hline \multicolumn{4}{|l|}{ LVH pattern } \\
\hline ASH & $25 \%$ & $57.3 \%$ & 0.004 \\
\hline Concentric & $44.2 \%$ & $30.1 \%$ & \\
\hline Apical & $28.8 \%$ & $11.7 \%$ & \\
\hline No hypertrophy & $1.9 \%$ & $1.0 \%$ & \\
\hline \multicolumn{4}{|l|}{ Echocanliographic characteristics } \\
\hline LVH (Sokolow \& Lyon) & $53.8 \%$ & $35.9 \%$ & 0.033 \\
\hline Left atrial enlargement & $44.2 \%$ & $49.5 \%$ & 0.534 \\
\hline Pathological 0 waves & $9.6 \%$ & $23.3 \%$ & 0.039 \\
\hline Left axis deviation & $11.5 \%$ & $17.2 \%$ & 0.270 \\
\hline Inverted T-waves & $82.7 \%$ & $69.9 \%$ & 0.086 \\
\hline $\mathrm{T}$-wave inversions in $\mathrm{V} 1-\mathrm{V} 4$ & $3.8 \%$ & $3.9 \%$ & 0.991 \\
\hline T-wave inversions in inferior leads & $1.9 \%$ & $5.8 \%$ & 0.269 \\
\hline T-wave inversions in lateral leads & $76.9 \%$ & $60.2 \%$ & 0.038 \\
\hline Deep T-wave inversions & $69.2 \%$ & $51.5 \%$ & 0.035 \\
\hline ST-segment elevation & $9.6 \%$ & $9.7 \%$ & 0.985 \\
\hline ST-segment depression & $50 \%$ & $35.0 \%$ & 0.071 \\
\hline
\end{tabular}

cardiac evaluation including 12-lead ECG and echocardiography Patients subject to therapeutic interventions potentially affecting repolarisation patterns were excluded.

Results Black patients revealed significantly different echocardiographic patterns of $\mathrm{LVH}$, with more concentric (44.2\% vs $30.1 \%$ ) and apical (28.8\% vs $11.7 \%$ ) hypertrophy compared to WP who exhibited more asymmetric septal hypertrophy (57.3\% vs $25.0 \%$ ) $(\mathrm{p}=0.004)$. Black patients exhibited a similar magnitude of $\mathrm{LVH}$ compared to WP $(17.3 \pm 4.9$ vs $18.8 \pm 4.1 \mathrm{~mm}, \mathrm{p}=0.069)$. Relating to ECG repolarisation abnormalities, BP exhibited more $\mathrm{T}$ wave inversions in the lateral leads $(76.9 \%$ vs $60.2 \%, p=0.038)$ and deep $(\geq-0.2 \mathrm{mV})$ T-wave inversions $(69.2 \%$ vs $51.5 \%, \mathrm{p}=0.035)$. Black patients also tended to display more ST segment depression $(50.0 \%$ vs $35.0 \%, \mathrm{p}=0.071$ ), although this was not statistically significant. In contrast, WP had significantly more pathological $\mathrm{Q}$ waves $(23.3 \%$ vs $9.6 \%, \mathrm{p}=0.039$ ).

Conclusions Ethnicity appears to exert a significant effect on ECG and echocardiographic patterns in patients with HCM. A significant proportion of black patients exhibit concentric LVH, highlighting the diagnostic challenges in distinguishing HCM from hypertensive heart disease and physiological adaptation to exercise in black individuals. The greater prevalence of deep $T$ wave inversions and $T$ wave inversions in the lateral leads underscores the importance of further evaluation of black individuals with such ECG repolarisation abnormalities, which may represent the initial expression of HCM

\section{THE RIGHT VENTRICLE OF THE ENDURANCE ATHLETE: THE RELATIONSHIP BETWEEN MORPHOLOGY AND DEFORMATION}

doi:10.1136/heartjnl-2011-300198.171

${ }^{1} \mathrm{D}$ Oxborough, ${ }^{2} \mathrm{R}$ Shave, ${ }^{3} \mathrm{G}$ Whyte, ${ }^{1} \mathrm{~K}$ Birch, ${ }^{4} \mathrm{~N}$ Artis, ${ }^{3} \mathrm{~K}$ George, ${ }^{5} \mathrm{~S}$ Sharma. ${ }^{1}$ University of Leeds, Leeds, UK; ${ }^{2}$ UWIC, Cardiff, UK; ${ }^{3}$ Liverpool John Moores University, Liverpool, UK; ${ }^{4}$ Leeds Teaching Hospitals NHS Trust, Leeds, UK; ${ }^{5}$ St Georges University Hospital, London, UK

Introduction It is well established that endurance exercise results in cardiac adaptation including eccentric hypertrophy of the left ventricle which can complicate the differential diagnosis of the athletic heart from some cardiac pathologies that may pre-dispose to sudden cardiac death. The impact of physiological conditioning on RV structure and function, and a similar diagnostic challenge with arrhythmogenic right ventricular cardiomyopathy (ARVC), has received less attention. A recent guideline paper from the American Society of Echocardiography (ASE) has provided a range for normal RV dimensions and functional deformation. These guidelines suggest the RV inflow (RVI) should be $<42 \mathrm{~mm}$ while the proximal outflow tract (RVOT) $<35 \mathrm{~mm}$. A recent paper also suggested that an RVOT dimension $>36 \mathrm{~mm}$ or $21 \mathrm{~mm} / \mathrm{m}^{2}$ is a major criterion for the diagnosis of ARVC and furthermore longitudinal RV deformation has been shown to be impaired in these patients. In view of this, the aims of this study are twofold:

1. To provide a range of absolute values for RV dimensions in 102 endurance athletes as well as providing a range of data indexed for body surface area (BSA).

2. To provide normal athlete data for indices of RV strain $(\varepsilon)$ and strain rate (SR)

Methods and Results One hundred and two (102) endurance athletes (86 males and 16 females) with a broad age range (mean \pm SD age (range) $=36 \pm 11(21-71)$ years) volunteered and were consecutively enrolled in the study. All subjects were either endurance runners or cyclists and were scanned at peak condition. Echocardiography provided measurements of RVI, RV length, RVOT and RV diastolic area (RVDarea). A $2 \mathrm{D}$ strain technique was utilised to provide indices of RVE and systolic and diastolic SR. The values for RVI 